\title{
Micro-MRI phenotyping of a novel double-knockout mouse model of congenital heart disease Jon O Cleary ${ }^{1}$, Karen McCue ${ }^{2}$, Anthony N Price*3, Sarah Beddow ${ }^{2}$, Roger J Ordidge ${ }^{4}$, Peter J Scambler ${ }^{2}$ and Mark F Lythgoe ${ }^{3}$
}

Address: ${ }^{1}$ Centre for Advanced Biomedical Imaging, Departments of Medicine and Medical Physics and Bioengineering, University College London, London, UK, ${ }^{2}$ Molecular Medicine Unit, UCL Institute of Child Health, University College London, London, UK, ${ }^{3}$ Centre for Advanced Biomedical Imaging, Department of Medicine and UCL Institute of Child Health, University College London, London, UK and ${ }^{4}$ Department of Medical Physics and Bioengineering, University College London, London, UK

* Corresponding author

from I3th Annual SCMR Scientific Sessions

Phoenix, AZ, USA. 21-24 January 2010

Published: 21 January 2010

Journal of Cardiovascular Magnetic Resonance 20 I0, I2(SuppI I):PI doi:I0.II86/I532-429X-I2-SI-PI

This abstract is available from: http://jcmr-online.com/content/I2/SI/PI

(c) 2010 Cleary et al; licensee BioMed Central Ltd.

\section{Introduction}

CHARGE and DiGeorge syndromes are conditions with incidences of 1 in 10,000 and 1 in 4000 and are strongly associated with haploinsufficiency of specific genes (CHD7 and TBX1). Both conditions are characterised by cardiovascular defects. Knockout mouse models are an important tool for the identification and characterisation of genes implicated in congenital heart conditions. MicroMRI is an emerging technique for high resolution cardiac phenotyping in a reduced time compared to conventional histology, enabling the acquisition of 3D images of multiple embryos in a single scan[1].

\section{Purpose}

Given the phenotypic overlap of these conditions we sought to examine the effect on cardiac morphology in double-knockout mouse embryos (Chd7+/-Tbx $\left.1^{+-}\right)[2]$, performing an initial assessment of these mice using MRI.

\section{Methods}

\section{Study Design}

18 embryos (1 wild-type, 7 Chd7+/-, 2 Tbx $1^{+/-}$and 8 Chd $\left.7^{+/-T b x} 1^{+/}\right)$were imaged and examined for cardiac abnormalities.

\section{Embryo Preparation}

16.5 dpc embryos were fixed for at least 2 weeks in a solution of $4 \%$ formaldehyde-PBS with $8 \mathrm{mM}$ Gd-DTPA
(Bayer-Schering AG) and then embedded in 1\% agarose gel (doped with $8 \mathrm{mM}$ Gd-DTPA) in $50 \mathrm{ml}$ centrifuge tubes.

\section{Imaging}

Performed on a Varian 9.4 T VNMRS system with $33 \mathrm{~mm}$ quadrature birdcage coil (RAPID Biomedical GmbH), using a $3 \mathrm{D}$ gradient echo sequence $(\mathrm{TE} / \mathrm{TR} / \mathrm{FA} / \mathrm{NSA}=9 /$
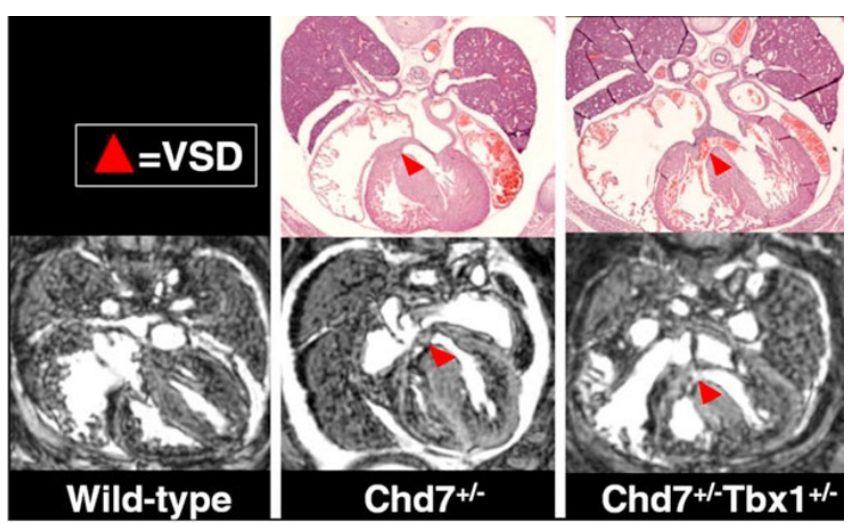

Figure I

Axial sections through example embryo datasets showing the presesnce of ventricular septal defects

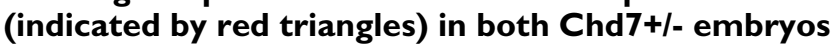
and double Chd7+/-TbxI+/- heterozygotes. 


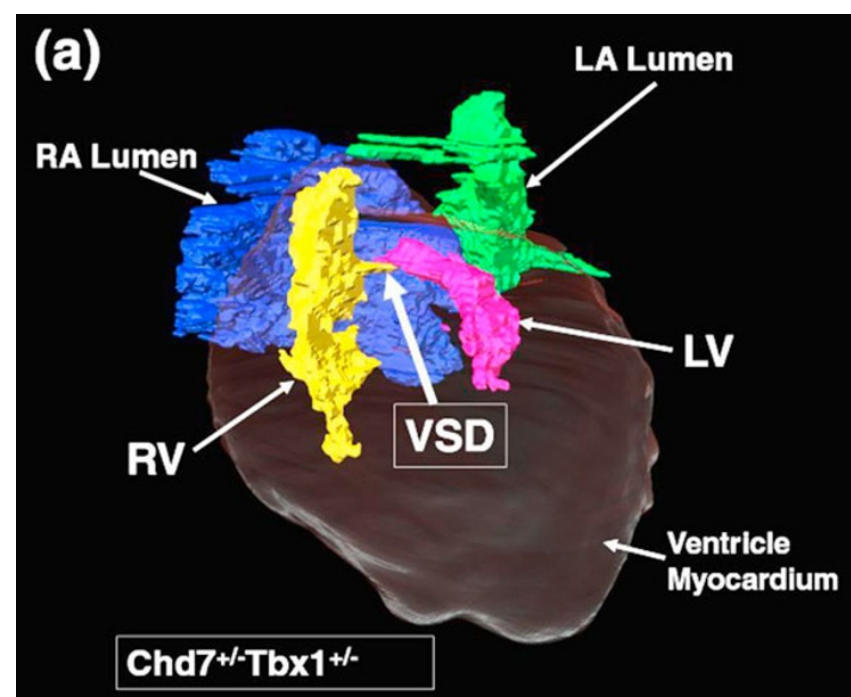

Figure 2

Volume rendering of a heart in a Chd7+/-Tbx I+/double heterozygous embryo. (RA: right atrium, RV: right ventricle, LA: left atrium, LV: left ventricle). A clear ventricular septal defect can be seen between left and right ventricles.

$20 / 60 / 7, \mathrm{FOV}=27 \times 27 \times 27 \mathrm{~mm}^{3}$, voxel size $=52 \times 52 \times$ $\left.52 \mu \mathrm{m}^{3}\right)$.

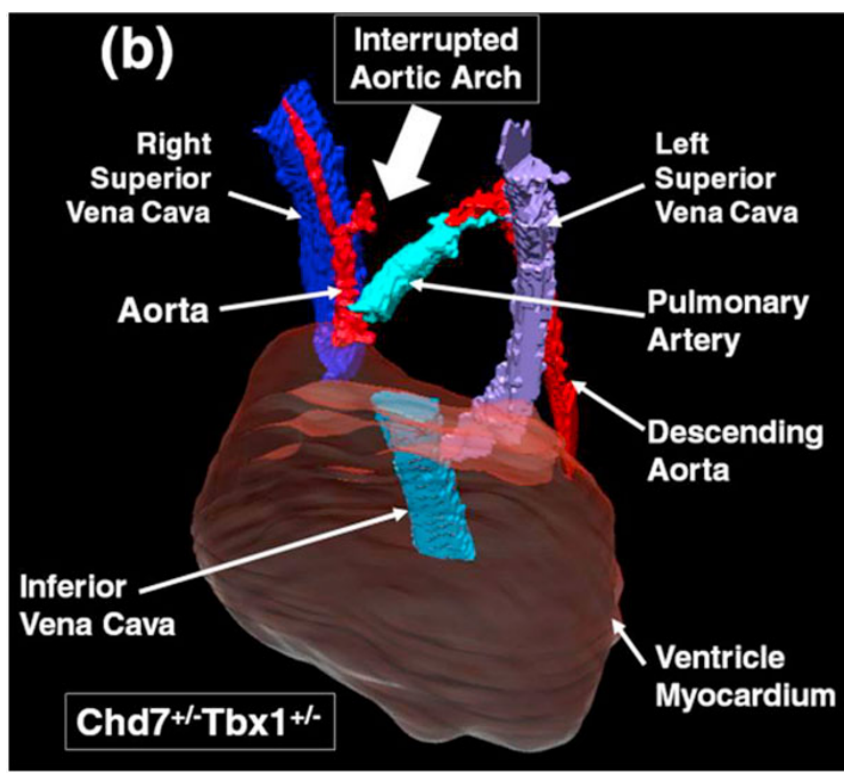

Figure 3

Volume rendering of the same embryo showing great vessel structures, An interrupted aortic arch was identified in this embryo (arrow).

\section{Image analysis}

Datasets were zero-filled to $26 \times 26 \times 26 \mu^{3}$ and reviewed in Amira (v5.2, Visage Imaging Inc.).

\section{Histology}

Embryos identified with abnormal hearts by MRI, were then histologically examined by H\&E staining.

\section{Results}

Of the 18 embryos scanned for MR analysis, we identified abnormal (thin or patent) ventricular septa (VSDs) in 6 embryos. Of these, 1 was a Chd $7^{+/}$-heterozygote (an incidence of $1 / 7$ in this study, see Fig. 1). VSDs were seen in 5/8 Chd7+/-Tbx1 embryos (Fig. 2). In one double heterozygote we also observed an interrupted aortic arch, in addition to a VSD, which was readily seen by MRI (Fig. 3).

\section{Conclusion}

Using micro-MRI we have successfully identified cardiac abnormalities in genetically-modified embryos. A single Chd7+/- embryo was found to have an abnormal ventricular septum. A relatively high incidence of VSDs was observed in Chd7 $7^{+/-T b x} 1^{+/-}$compared to Chd7 $7^{+/-m i c e,}$ indicating possible interaction of these two genes. An interrupted aortic arch was identified in one $\mathrm{Chd} 7^{+/}$ Tbx1+/- embryo. All abnormal findings were later confirmed by histology which indicates that micro-MRI is an effective technique for cardiac phenotyping.

\section{References}

I. Cleary JO, Price AN, et al.: NMR Biomed 2009, 22(8):857-866.

2. McCue K, Randall V, et al.: 06-P038. Mech of Dev 2009, I 26:SI3I. 